\title{
Clinical Comparative Study on the Treatment of Inferior Pole Fractures of Patella by Double-row Anchor Suture Bridge Technique Combined with Kirschner Wire Tension Band Internal Fixation
}

\author{
chen bingqian ( $\nabla$ lemonch@163.com )
}

Changshu Hospital Affiliated to Soochow University: First People's Hospital of Changshu City https://orcid.org/0000-0002-4012-3481

\section{zhengfei wang}

Changshu Hospital Affiliated to Soochow University: First People's Hospital of Changshu City zhi chen

Changshu Hospital Affiliated to Soochow University: First People's Hospital of Changshu City xiaohong qu

Changshu Hospital Affiliated to Soochow University: First People's Hospital of Changshu City xiaowen fang

Changshu Hospital Affiliated to Soochow University: First People's Hospital of Changshu City

xuesong wang

Affiliated Hospital of Jiangnan University

guoxiu KE

Changshu Hospital Affiliated to Soochow University: First People's Hospital of Changshu City

\section{Research Article}

Keywords: inferior pole fractures of patella, suture anchor, suture bridge technique, Kirschner wire tension band internal fixation

Posted Date: January 13th, 2022

DOI: https://doi.org/10.21203/rs.3.rs-1215918/v1

License: (1) This work is licensed under a Creative Commons Attribution 4.0 International License. Read Full License 


\section{Abstract}

Objective: To investigate the method, technique and clinical efficacy of double-row anchor suture bridge technique in the treatment of inferior pole fractures of patella, and to compare it with traditional Kirschner wire tension band internal fixation.

Methods: General information A total of 62 patients were enrolled in this study, including 35 males and 27 females, aged 31-80 years old, with an average of (53.2 \pm 4$)$ years old. All the patients were divided into two groups by a random double-blind method: Group A (double-row anchor suture bridge technique group) and Group B (Kirschner wire tension band internal fixation group), with 32 cases and 30 cases in each group, respectively. The two techniques were performed by the same group of doctors to fix the fracture respectively, and the intraoperative bleeding, operation time, incidence of postoperative complications, knee function score, etc., were compared between the two techniques.

Results: Group A (double-row anchor suture bridge technique group): operation time 56-80min, with an average of $(60 \pm 10) \mathrm{min}$; intraoperative blood loss $30-56 \mathrm{ml}$, with an average of $(43 \pm 10) \mathrm{ml}$. All incisions healed in one stage and stitches were removed after 2 weeks. No complications such as incision infection, flap necrosis and nerve injury occurred. The average healing time of the fracture was 12 weeks. At the last follow-up, the Bostman score was (28.5 \pm 3.3$)$, and the excellent rate was $93.7 \%$. Group B (Kirschner wire tension band internal fixation group): operation time 54-76min, with an average of $(56 \pm 10) \mathrm{min}$; intraoperative blood loss $31-50 \mathrm{ml}$, with an average of $(43 \pm 80) \mathrm{ml}$. One patient developed delayed incision healing postoperatively. Four patients experienced Kirschner wire withdrawal, one of which was accompanied by infection. Two patients were found to have mild separation of distal fracture fragments, despite the fracture being fine. The average healing time of the fracture was 13 weeks. At the

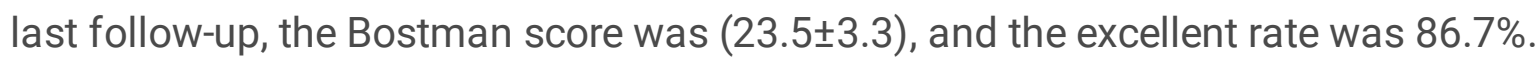

Conclusion: Double-row anchor suture bridge technique is applied to inferior pole fractures of patella by virtue of its various effects, such as the complete preservation of the inferior pole fragments during the operation, satisfactory fracture reduction, firm fixation, and meeting patients' requirements for early postoperative ambulation. This technology is superior to traditional Kirschner wire tension band internal fixation owing to its advantages of no need to take out the internal fixation, firm fixation, no need to worry about Kirschner wire withdrawal and fewer complications, and its clinical efficacy satisfaction rate is also better than traditional surgical methods. In short, double-row anchor suture bridge technique is an ideal surgical procedure for the treatment of the inferior pole fracture of patella with safety, reliability and high satisfaction.

\section{Background}

Inferior pole fractures of patella are common intra-articular fracture with small fracture fragments and mostly comminuted in clinic, which is a difficult point in fracture treatment. Currently, wire tension band fixation and resection of the inferior pole of patella, etc. are the preferred surgical treatments for inferior 
pole fractures of patella. Each treatment has its own advantages and disadvantages, and reports on its efficacy are inconsistent. Resection of the inferior pole of patella is rarely applied at present because it changes the biomechanical characteristics of the patella, causes weakened quadriceps muscle strength and patellofemoral arthritis, and affects the function of the knee joint. In contrast, Kirschner wire tension band internal fixation is widely applied. In this method, the wire is placed on the tension side of the patella, and the tension it is subjected to will change into the compressive stress at the fracture end during knee flexion, promoting the fracture healing. In the traditional $\mathrm{AO}$ tension band technique, two Kirschner wires are fixed in the center of the bone, and the " 8 "-shaped steel wire is fixed at both ends of the Kirschner wires. It is reported in the literature that although the tension band wire fixation method is reliable and achieves satisfactory clinical outcomes, it also has numerous drawbacks ${ }^{[7]}$ : Not suitable for small and comminuted bones; Kirschner wires can pierce the skin and cause painful infections; Kirschner wire needs to be removed by a second operation.

Suture anchor technique has also been applied in the treatment of inferior pole fractures of patella as it continues to develop. It is superior to wire tension band fixation and resection of the inferior pole of patella thanks to its advantages of retaining fracture fragments of the inferior pole of patella, no need to take out the internal fixation, bone-bone healing superior to tendon-bone healing, and no Kirschner wire withdrawal. Nevertheless, traditional anchor technique alone is unable to provide sufficient stability for the fractured end, and patients often need to be protected by a brace postoperatively. Moreover, active knee joint functional exercises cannot be performed early, and the reduction of comminuted fractures is not ideal.

To overcome the shortcomings of traditional surgical methods, innovation and improvement have been made on the double-row anchor suture bridge technique in rotator cuff repairs, which is applied to the treatment of inferior pole fractures of patella. Specifically, after the fracture fragments and ligaments are sutured by the internal-row of anchors, the sutures are compressed on the surface of the patella by the external-row of extrusion nails, similar to a parachute bag, thus enhancing the stability of the fracture without the need for secondary removal of internal fixation, which has significant advantages. A review of the literature found no reports of a similar technique in the treatment of inferior pole fractures of patella. From January 2019 to August 2020, the double-row anchor suture bridge technique was used by our hospital for the treatment of inferior pole fractures of patella, and compared with the traditional Kirschner wire tension band internal fixation, providing a basis for the selection of clinical surgical methods. The specific details are reported as following.

\section{Materials And Methods}

General information: A total of 62 patients were enrolled in this study, including 35 males and 27 females, aged $31-80$ years old, with an average of $(53.2 \pm 4)$ years old. All the patients were divided into two groups by a random double-blind method: Group A and Group B. 
Group A (double-row anchor suture bridge technique group): 32 cases, including 19 males and 13 females, aged 31-72 years old, with an average of (51.3 \pm 5$)$ years old. Among them, there were 17 cases of right fractures and 15 cases of left fractures, all of which were closed fractures. Injury causes: 28 cases of fall injury, 4 cases of car accident injury.

Group B (wire tension band group): 30 cases, including 16 males and 14 females, aged 39-80 years old, with an average of (52.1 \pm 2 ) years old. Among them, there were 14 cases of right fractures and 16 cases of left fractures, all of which were closed fractures. Injury causes: 27 cases of fall injury, 3 cases of car accident injury.

Inclusion criteria: inferior pole fractures of patella, closed fracture.

Exclusion criteria: fractures involving articular surfaces, open fractures, and local infectious lesions.

All patients underwent routine X-ray and CT scan for three-dimensional reconstruction. After admission, patients were given ice compress, temporary immobilization of brace, and pain relief. Surgery is performed after completion of the intraoperative examination, usually 3-4 days after the injury.

\subsection{Surgical methods}

Group A (double-row anchor suture bridge technique): After satisfactory anesthesia, routine disinfection was performed. An incision was made layer by layer to reveal the inferior pole fractures of patella and the patellar ligament. Clean up the hematoma and soft tissue of the fractured end. At the proximal end of the patella fracture, two suture anchors (one medial and one lateral) were screwed close to the articular surface of the patella, each with two sutures. Kirschner wire was utilized to drill longitudinally at the inferior pole fractures of patella, and the patellar ligament was sutured with transverse mattress suture for several stitches through both ends of the anchor suture line. After reduction and tightening of the fractured end, a total of 4 knots were tied. Subsequently, four knotted tail wires were gathered to cover the bone fragments of the inferior pole of the patella, and then passed through a Johnson \& Johnson Versalok extrusion anchor to drive it into the bone at the upper end of the patella. Finally, X-ray fluoroscopy confirmed that the fracture was ideally reduced, the knee joint was moved, and the fracture was fixed firmly. See Figure 1.

Group B (Kirschner wire tension band internal fixation): After successful anesthesia, a conventional disinfection towel was used to take a longitudinal incision of the patella and cut it layer by layer to fully expose the inferior pole of the patella and the patellar ligament. Hematoma and soft tissue embedded in the fractured end of the fracture was removed, and the fracture of the inferior pole of the patella was reduced and temporarily fixed with reduction forceps. After X-ray fluoroscopy confirmed a satisfactory reduction of the fracture, two Kirschner wires were drilled from the inferior pole of the patella to the body of the patella and out from the proximal end of the patella. A wire ring with a needle was used to tie the edge of the patella, and another wire with an " 8 "-shaped steel wire was used to tie the edge of the patella 
to tighten the wire. Fluoroscopic X-ray showed a satisfactory reduction of fracture, the knee joint was moved, and the fracture fragments were firmly fixed. Finally, the incisions were rinsed and sutured layer by layer.

\subsection{Postoperative management and follow-up}

Both groups were given ice compress, pain relief and swelling treatment postoperatively. On the first day postoperatively, patients were able to do functional exercises of the knee and ankle, and to perform functional exercises of the knee on a CPM machine from $45^{\circ}$ to $120^{\circ}$ three times a day for half an hour each time. On the third day postoperatively, patients were able to move with crutches out of bed. Three months postoperatively, patients resumed their daily activities. X-ray examinations were performed on the patients in January, March, June, and December to find out the fracture healing.

The postoperative incision healing and postoperative complications were recorded, and the knee joint function score was recorded as well. The efficacy evaluation criteria of Bostman patella fracture were utilized to score the patella fracture in terms of range of motion, duration of motion, pain and atrophy: excellent (28-30 points), good (20-27 points), poor (<20 points).

\subsection{Statistical analysis}

All data in this study were statistically analyzed using SPSS 19.0 software. Measurement data were expressed as $(x \pm s)$, and paired sample $T$ test was used for comparison. The level of significance was set at $\mathrm{P}<0.05$.

\section{Results}

In Group A, the operation time was 56-80 min, with an average of $(60 \pm 10) \mathrm{min}$, and the intraoperative blood loss was 30-56 ml, with an average of $(43 \pm 10) \mathrm{ml}$. All incisions healed in one stage and stitches were removed after 2 weeks. No complications such as incision infection, flap necrosis and nerve injury occurred. Patients in this group were followed up for 10-18 months, with an average follow-up of 12 months. All fractures healed in 10-20 weeks, with an average healing time of 12 weeks. At the last followup, the Bostman score was (28.5 \pm 3.3$)$, excellent in 30 cases and good in 2 cases, with an excellent rate of $93.7 \%$. The range of motion of the knee joint was $(-1.5 \pm 2.0)^{\circ}$ when the knee was extended and $(125 \pm 5.0)^{\circ}$ when the knee was bent. The muscle strength of quadriceps femoris was grade 5 .

In Group B, the operation time was $54-76 \mathrm{~min}$, with an average of $(56 \pm 10) \mathrm{min}$, and the intraoperative blood loss was $31-50 \mathrm{ml}$, with an average of $(45 \pm 80) \mathrm{ml}$. One patient developed delayed incision healing postoperatively, and the bacteria culture was negative. Incision healing occurred 3 weeks after intensive dressing change. Four patients had the withdrawal of the Kirschner wire, which pushed up against the skin and caused pain, and one of the patients had Kirschner wire piercing through the skin, accompanied 
by infection. Three patients took out the Kirschner wires that had been withdrawn in the outpatient operating room, and the rest of the internal fixation was kept until the fracture healed before being taken out. Patients with infection caused by withdrawal of Kirschner wire were given enhanced dressing change, and local infection was controlled after intravenous administration of sensitive antibiotics. All patients took out all internal fixations 1 year postoperatively. Two patients were found to have mild separation of inferior fracture fragments, both of which had small and comminuted fractures, despite the fracture being fine.

Patients in this group were followed up for 11-17 months, with an average follow-up of 12 months. All fractures healed in 11-21 weeks, with an average healing time of 13 weeks. At the last follow-up, the Bostman score was (23.5 \pm 3.3$)$, excellent in 26 cases and good in 4 cases, with an excellent rate of $86.7 \%$. The range of motion of the knee joint was $(-1.2 \pm 1.5)^{\circ}$ when the knee was extended and $(122 \pm 3.0)^{\circ}$ when the knee was bent. The muscle strength of quadriceps femoris was grade 5 .

\begin{tabular}{|c|c|c|c|c|c|}
\hline \multicolumn{6}{|c|}{ Table 1} \\
\hline \multicolumn{6}{|c|}{ Personal information and rates of excellent results in both groups } \\
\hline Group & $\mathrm{n}$ & Mean age & Mean operation time & Mean bleeding & Rates of excellent results (\%) \\
\hline $\mathrm{A}]$ & 32 & $₫ 51.3 \pm 5 \rrbracket$ & $\nabla 60 \pm 10 \rrbracket$ & $₫ 43 \pm 10 \rrbracket$ & 93.7 \\
\hline $\mathrm{B} \square$ & 30 & $\triangle 52.1 \pm 2 \rrbracket$ & $ه 56 \pm 10 \rrbracket$ & $₫ 45 \pm 80 \rrbracket$ & 86.7 \\
\hline
\end{tabular}

\begin{tabular}{|lllll|}
\hline \multicolumn{5}{|c|}{ Complications in both groups } \\
Group & $\begin{array}{l}\text { Number of } \\
\text { infections }\end{array}$ & $\begin{array}{l}\text { Loose internal } \\
\text { fixation }\end{array}$ & $\begin{array}{l}\text { Re displacement of } \\
\text { fracture }\end{array}$ & $\begin{array}{l}\text { Remove the internal } \\
\text { fixation }\end{array}$ \\
\hline A $\square$ & 0 & 1 & 0 & 0 \\
\hline B & 1 & 4 & 2 & 30 \\
\hline
\end{tabular}

\section{Discussion}

\subsection{Characteristics of inferior pole fractures of patella and disadvantages of traditional surgical methods}

Inferior pole fractures of patella are clinically common, accounting for approximately $9.3 \%-22.4 \%$ of all patella fractures ${ }^{[1]}$. It has the following characteristics: Firstly, the fracture fragments are small, and most of them are comminuted; Secondly, the inferior part of the patella is the insertion point of the patellar 
tendon, which is subjected to greater tensile stress. Therefore, the fracture is under a certain degree of particularity and difficulty in reduction and fixation ${ }^{[2][3][9]}$.

So far, wire tension band fixation and resection of the inferior pole of patella, etc. are the preferred surgical treatments for inferior pole fractures of patella ${ }^{[16]}{ }^{[17]}$. Resection of the inferior pole of patella is rarely applied at present because it changes the biomechanical characteristics of the patella, causes weakened quadriceps muscle strength and patellofemoral arthritis, and affects the function of the knee joint.

In contrast, Kirschner wire tension band internal fixation is widely applied. In this method, the steel wire is placed on the tension side of the patella, and the tension it is subjected to will change into the compressive stress at the fracture end during knee flexion, promoting the fracture healing ${ }^{[20]}$. In the traditional AO tension band technique, two Kirschner wires are fixed in the center of the bone, and the " 8 "shaped steel wire is fixed at both ends of the Kirschner wires. It is reported in the literature that although the tension band wire fixation method is reliable and achieves satisfactory clinical outcomes, it also has numerous drawbacks ${ }^{[7]}$. First of all, this technique is not suitable for small bones; Secondly, for comminuted fractures, the fixation technique is not reliable; In addition, Kirschner wire tension band is prone to kirschner wire withdrawal, which can compress the skin and cause pain, or even pierce the skin. Finally, Kirschner wire tension band must be removed by a second operation, contributing to increased patient pain and medical costs ${ }^{[8]}$. In this study, Kirschner wire withdrawal occurred in 4 cases in the wire tension band group, accounting for $13 \%$ of all cases, with one case piercing the skin and developing infection. In this group, two cases had fracture displacement. Despite eventual bone healing, the inferior pole of the patella became longer, which is bound to change the biomechanics of the knee joint and affect the function of the knee joint.

\subsection{Advantages and disadvantages of traditional anchor technique}

Suture anchor was first applied in the treatment of rotator cuff tears and gradually applied in the treatment of inferior pole fractures of patella with the development of techniques in orthopaedic ${ }^{[13][14]}$. Fixation of comminuted fractures of the inferior pole of the patella with suture anchors can not only restore the integrity of the knee extension device, but also preserve the fracture of the inferior pole of the patella. Meanwhile, bone-bone healing is also superior to tendon-bone healing. Finally, suture anchor does not need to be removed, avoiding a secondary operation ${ }^{[16]}$. In short, this technique has obvious advantages over traditional wire tension band internal fixation and resection of the inferior pole of patella.

The traditional suture anchor method is a single row anchor fixation, which also has many drawbacks ${ }^{[17]}$ [18]. First of all, single-row anchors rely only on sutures, which cannot provide sufficient stability at the fracture end and prevent early ambulation postoperatively. Moreover, for comminuted fractures, fracture 
fragments cannot be reduced well by wire anchors alone, which may easily cause displacement of fracture fragments.

\subsection{Advantages of double-row anchor suture bridge technique}

"Double-row anchor suture bridge technique" is a novel technique for arthroscopic repair of rotator cuff tears ${ }^{[19]}$, which was first proposed by Park in $2006^{[12]}$. Firstly, the sutures of the internal-row anchors passed through the tendon in a horizontal mattress and were knotted. After passing the suture tail through the external-row pressing screw, the external-row nails were inserted into the greater tubercle of the humerus at the lateral insertion point of the rotator cuff, so as to complete the uniform extrusion of the rotator cuff by the "suture bridge". This technique is more stable than the traditional anchor fixation technique and has greater anti-pulling strength. Furthermore, due to the extrusion pressure of the "suture bridge" on the rotator cuff, the contact surface between tendon and bone is increased and the tendon and bone healing is promoted. In view of the advantages of suture bridge technique, this technique has gradually been widely applied in shoulder surgery, and its scope of application has also been expanded to be used in the treatment of some fractures. It has been reported in literature ${ }^{[14][15]}$ that some scholars have applied this technique to the treatment of avulsion fractures of the greater tuberosity of humerus and intercondylar spine avulsion fractures of the tibia, achieving satisfactory clinical results. However, there are no reports about the application of this technique in the treatment of patellar fractures.

In order to overcome the shortcomings of the traditional suture anchor method, we modified the technique of "double-row anchor suture bridge" in shoulder arthroscopy and applied it to the treatment of the fracture of the inferior pole of patella. Specifically, two internal row anchors were screwed into the proximal end of the patella fracture, and the sutures were passed through the inferior pole fracture fragments via the bone tunnel, and the patellar ligament was sutured with horizontal pad and knotted. The tail line of the suture was arranged to cover the bone fragments at the lower part of the patella. After passing the tail of the suture through the external extrusion anchor, the external extrusion nail was driven into the upper part of the patella (see Figures A-B). This technology not only strengthens the stability of the fracture, but also gathers the lower pole fragments and maintains the reduction of the fracture. Since the sutures of the anchors wrap around the bone fragments below the patella in the shape of a parachute, we also call it "parachute technique". This technique was used to treat patella fractures of the lower pole and was compared with traditional Kirschner wire tension band fixation. No significant difference was found between the two in terms of operation time and surgical bleeding, but the doublerow anchor suture bridge technology group did not experience internal fixation withdrawal or skin penetration, while 4 cases in the control group had such circumstances, and 1 case was infected as a result. The suture bridge group is more reliable in fixation and can be applied to small and crushed bones. In this group of cases, the suture bridge technique group did not have any loss of fracture at the later stage of reduction, while the control group had 2 cases of loss of fracture during late reduction, despite 
the fracture being healed. Finally, the excellent rate of the knee joint function score (Bostman score) of the suture bridge technique group was also higher than that of the traditional technique group.

Patients in the double-row anchor suture bridge technique group were able to perform early knee flexion and extension exercises postoperatively, and no internal fixation loosening or fracture re-displacement were found. All fractures were osseous union, and satisfactory clinical effects were achieved.

\subsection{Points of attention and surgical experience}

First of all, the two anchored implant points should be lower than the center of the patella section, close to the subchondral bone, and need to be screwed to a sufficient depth in order to achieve the maximum holding force. For patients with relatively loose bone, the screw can be screwed deeper until it approaches or even breaks through the cortical bone at the upper end of the patella to increase the holding force of the anchor. If the anchor is found to be loose when screwed in, and it is estimated that sufficient stability cannot be obtained, it is necessary to replace or assist other internal fixation methods. In the initial stage of the operation, one case was found to have a loose screw anchor during the operation, and other internal fixation methods were replaced in time. In the coronal position, the two anchors should be positioned equally on either side of the midline of the patella to facilitate fracture reduction and stress distribution. All the patients in this group were operated on according to the above methods, and no loosening of anchor or loss of fracture block reduction was found.

Secondly, after the suture passes through the fracture of the lower pole, one end of the tail line needs to be sewed into the patellar ligament for 2-3 stitches and then tied into the other end for fixation. Direct knotting may result in cutting of bone, especially in comminuted fractures. After all the four wires are knotted, the position of the tail wire should be adjusted, and the broken bones of the bottom pole should be covered as much as possible, resembling a parachute bag, so as to achieve the optimal fixing effect. All cases in this group were performed according to the above methods, and no tendon cutting or loss of fracture block reduction was found.

Moreover, intraoperative fluoroscopy is required to ensure that the extrusion screw is in the center of the upper pole of the patella when the external extrusion screw is inserted, and attention should be paid to the matching perforator to open the bone canal. In case of too small perforator and insufficient bone canal diameter, splitting of the bone canal wall may occur when the extrusion anchors are inserted, and in severe cases, iatrogenic fractures may occur. Meanwhile, proper tail line tension needs to be adjusted for driving the extrusion anchors. Too little tension may make it impossible to achieve favourable fixation; Too much tension may result in the cutting of the bone tunnel wall with sutures and displacement of the inferior pole fracture.

Finally, after the fracture is fixed, the two patellas are circulated with non-absorbable ETHIBOND suture to strengthen the fixation. Bend and extend the knee joint to confirm that the fractured end is firmly fixed and not separated. If instability is found, a brace is recommended for postoperative protection. 


\section{Conclusions}

Double-row anchor suture bridge technique is applied to inferior pole fractures of patella by virtue of its various effects, such as the complete preservation of the inferior pole fragments $₫$ satisfactory fracture reduction, firm fixation, and meeting patients' requirements for early postoperative ambulation. This technology is superior to traditional Kirschner wire tension band internal fixation owing to its advantages of no need to take out the internal fixation, firm fixation, no need to worry about Kirschner wire withdrawal and fewer complications, and its clinical efficacy satisfaction rate is also better than traditional surgical methods. In short, double-row anchor suture bridge technique is an ideal surgical procedure for the treatment of the inferior pole fracture of patella with safety, reliability and high satisfaction.

\section{Declarations}

\section{Ethics approval and consent to participate:}

The experimental protocol was established, according to the ethical guidelines of the Helsinki Declaration and was approved by the Human Ethics Committee of First Peoples' Hospital of Changshu City. Written informed consent was obtained from individual or guardian participants.

\section{Consent for publication:}

All the authors are consent to publish the article

\section{Data Availability Statement:}

All data generated or analysed during this study are included in this published article (and its supplementary information files).

\section{FUNDING:}

There is no funding for this study

\section{Competing interests:}

We declare that they have no competing interests.

\section{The authors' contributions in the article:}


Conceived and designed the experiments: Chen Bing-qian, Ke guoxiu

Performed the surgeries: Chen Bing-qian, Qu Xiaohong Fang Xiao-wen

Data acquisition and analysis: Chen Bing-qian, Chen zhi.

Wrote the manuscript: Chen Bing-qian, Wang zhengfei

\section{Acknowledgements:}

First, I would like to show my deepest gratitude to my supervisor, Dr. Qirong Dong, a respectable, responsible and resourceful scholar. I shall extend my thanks to Mrs. GE for all her kindness and help. Last but not least, I' d like to thank all my friends for their encouragement and support.

We make a statement that all authors have agreed to the submission to the journal and that the manuscript is not currently under submission in any other journal.

\section{References}

1. Henrichsen JL, Wilhem SK,Siljander MP,et al. Treatment of patella fractures[J]. Orthopedics. 2018;41(6):e747-55.

2. Sigvard T. Rockwood and green's fractures in adults. 6th ed[J]. Jbjs,2007,89:511.

3. Li L, Haichao H, Zhong L, et al. Modified patella tibial tuberosity cerclage technique for the treatment of the lower pole of the patella[J]. Chinese Journal of Bone Joint Injury. 2014;29(S1):79.

4. Tian Jian Xu, Yajun C, Zheng, etc. Effectiveness analysis of limited small incision with simple Krackow suture in treatment of acute closed Achilles tendon rupture[J]. Chinese Journal of Reconstructive and Reconstructive Surgery, 2017, 31(11):1322-1325.

5. Lysholm J, Gillguist J. Evaluation of knee ligament surgery results with special emphasis on use of ascoring scale[J]. Am J SportsMed. 1982;10(3):150-4.

6. Lu Tianxiang Y, Hua. Chen Zunrong, etc. A case report of repair, reconstruction and rehabilitation of patellar tendon rupture of high-level long jump athletes[J]. Chinese Journal of Sports Medicine. 2016;35(1):71-3.

7. Ma Xiangyu X, Liangbi L, Bing Z, Dapeng. Progress in the treatment of partial resection of fractures of the lower pole of the patella[J]. Clinical Journal of Medical Officers. 2020;48(04):478-80.

8. Chen Nong L, Zhi D, Jian, et al. Application of suture anchors in the treatment of ankle deltoid ligament injury[J]. Chinese Journal of Bone Joint Injury. 2011;26(7):235-6.

9. Khazen GE, Wilson AN, Ashfap S, et al. Fixation of calcaneal avulsion fractures using screws with and without suture anchors: a biome-chanical investigation[J]. Foot Ankle Int. 2007;28(11):1183-6. 
10. Gu Deshuai Z, Ren Yu, Wenjun Z. Liwen. Clinical application of absorbable net-sliding intertexture with tension band wiring comminuted fracture of inferior patella pole[J]. China Journal of Orthopaedics Traumatology. 2018;31(10):903-6.

11. Shi Dufang W, Dongmei, et al. Tensile and Viscoelastic Properties of Chinese Patellar Ligaments: Preliminary Comparison between Chinese and Westerns[J]. Journal of Chinese Biomedical Engineering, 2010, 4(2): 314-320.

12. Wang Peizhao H, Xu W, Jinyang XY, Yanhao Y, Honglue T. Clincal observation of non-absorbable suture cerclage combined with patella claw fixation for treatment of inferior patella fractures[J]. Chinese Journal of Reconstructive Reconstructive Surgery. 2020;34(10):1243-7.

13. Zhang Dabao M, Bo, Jinping L. Krachow method suture combined with patella tibial tension band to treat lower pole fracture of patella[J]. Practical Clinical Journal of Integrated Traditional Chinese Western Medicine. 2019;19(02):78-9.

14. Zhang Liang Z, Zandong K, Xin R, Bo ZX, Jiang Z. Arthroscopic suture bridge technique for elderly patients with shoulder dislocation combined with supraspinatus avulsion fracture[J]. Orthopedics. 2020;11(06):475-9.

15. Li Ji L, Weixiong ZL, Hao Z, Ketao W, Yimeng Y. Clinical research on suture anchor fixation for the treatment of avulsion fracture of tibial intercondylar eminence under arthroscopy[J]. China Journal of Orthopaedics Traumatology. 2017;30(11):1023-8.

16. Cho JW, Kim J, Cho WT, et al. Comminuted inferior pole fracture of patella can be successfully treated with rim-plate-augmented separate vertical wiring[J]. Arch Orthop Trauma Surg. 2017;138(Suppl 2):1-8.

17. Keun $\mathrm{H}$, Song, Hyun J, et al. Separate vertical wiring for the fixation of comminuted fractures of the inferior pole of the patella.[J]. Yonsei medical journal; 2014.

18. Fan $M$, Wang $D$, Sun $K$, et al. Study of double button plate fixation in treatment of inferior pole of patella fracture[J]. Injury, 2020, 51(3).

19. Kim KS, Suh DW, Park SE, et al. Suture anchor fixation of comminuted inferior pole patella fracturenovel technique: suture bridge anchor fixation technique[J]. Archives of Orthopaedic and Trauma Surgery, 2020:1-9.

20. Misir A, Kizkapan TB, Uzun E, et al. Fracture Patterns and Comminution Zones in OTA/AO 34C Type Patellar Fractures[J]. Journal of Orthopaedic Trauma, 2019:1.

\section{Figures}




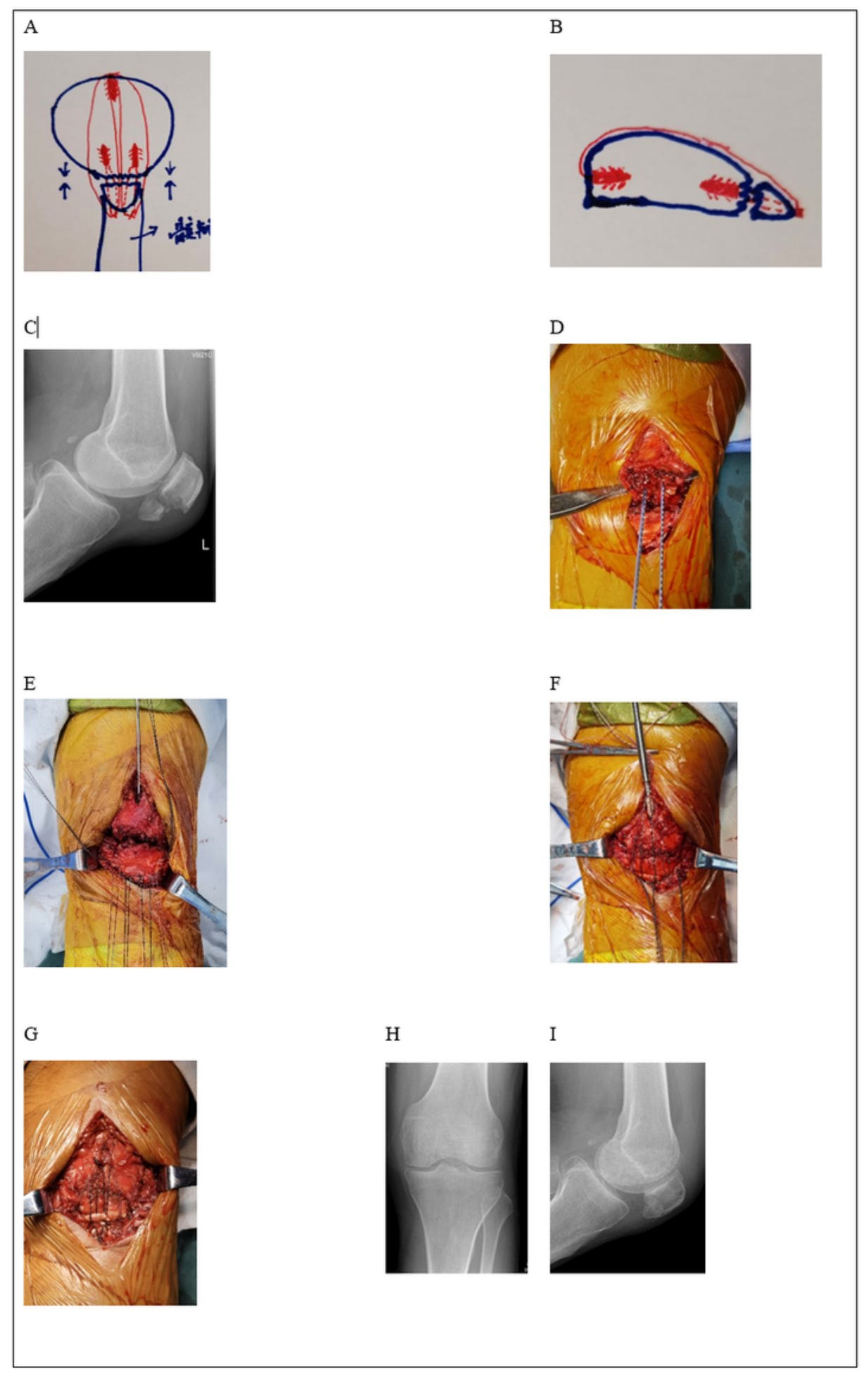

\section{Figure 1}

A-B. Freehand sketching of double-row anchor suture bridge technique for treatment of inferior pole fractures of patella: Two internal-row anchors were driven into the proximal end of the patella fracture, and the sutures passed through the inferior pole of the fracture fragments via the bone tunnel. The patellar ligament was sutured in a horizontal mattress and knotted, and the tail line of the sutures was 
arranged to cover the bone fragments at the inferior pole of the patella. After passing the tail of the sutures through the external-row anchors, the external-row anchor was nailed on the top of the patella.

Typical case: A 58-year-old male patient was admitted to the hospital due to pain in the left knee joint caused by trauma and limited movement for 1 hour. Figure C: X-ray shows a fracture of the inferior pole of the left patella with an obvious displacement of the fractured end. Figures D-G: Open reduction and double-row anchor of inferior patella fracture under epidural anesthesia. Figure $\mathrm{H}$ : Radiographically confirmed ideal reduction of the fracture. Figure I: One year postoperatively, X-ray radiographs showed good fracture union and no obvious fracture displacement. 